\title{
Nuclear equation of state from the nonlinear relativistic mean field theory
}

\author{
B. M. Waldhauser, J. A. Maruhn, H. Stöcker, and W. Greiner \\ Institut für Theoretische Physik der Johann Wolfgang Goethe Universität, \\ D-6000 Frankfurt am Main, Federal Republic of Germany
}

(Received 29 September 1987)

\begin{abstract}
The properties of symmetric nuclear matter are investigated in the nonlinear relativistic mean field theory of nuclear matter. We consider the constraints imposed by four nuclear ground state properties on the coupling constants and on the equation of state at zero and at finite temperature. We find that the compression constant $K\left(\rho_{0}\right)$ as well as the temperature is irrelevant for the stiffness of the equation of state for $m^{*}\left(\rho_{0}\right) \leq 0.7$. The main point is that the relativistic mean field theory exhibits acausal and unphysical behavior for compressibilities below $K\left(\rho_{0}\right)=200 \mathrm{MeV}$. Every set of coupling constants with a negative quartic coupling constant $c$ is unstable against small quantum fluctuations.
\end{abstract}

\section{INTRODUCTION}

One of the central aims of high-energy heavy-ion physics is to determine the equation of state of nuclear matter at all physically interesting densities. ${ }^{1}$ Thus far the equation of state is known at only one density value. This can be inferred from the structure of finite nuclei. It reveals that nuclear matter saturates at a density of about $\rho_{0}=0.15 / \mathrm{fm}^{3}$ with a binding energy per particle $E / A\left(\rho_{0}\right)=-16 \mathrm{MeV} .{ }^{2}$ In the following we adopt these values. Analysis of the nuclear monopole vibrations seemed to infer the compression constant to be $K\left(\rho_{0}\right)=210 \pm 30 \mathrm{MeV},{ }^{3}$ but recent calculations yield compressibilities $K\left(\rho_{0}\right) \simeq 100 \mathrm{MeV}$ unreasonably low (Ref. 4) or higher values, $K\left(\rho_{0}\right) \simeq 344 \mathrm{MeV}, K\left(\rho_{0}\right)$ $=290 \pm 20 \mathrm{MeV}$ (Ref. 5). Therefore, it seems that the question of determining the compression constant is still completely open. A similar problem occurs for the effective nucleon mass $m^{*} / m$ at $\rho_{0}$ : Values from 0.6 to $0.9 \mathrm{~m}$ are being deduced from the energy dependence of the proton-nucleus optical potential.

Any reasonable theory of nuclear matter must either predict these four properties of nuclear matter from first principles or else incorporate them into a self-consistent phenomenological approach. These four quantities determine the essential properties of nuclear matter and nuclear structure in the one-particle sector. ${ }^{6}$ An efficient parametrization of these quantities in a relativistic field theoretic framework is therefore desirable, because then one can study the properties of nuclear matter as a function of any of the above quantities. This parametrization can be done in a theory that is renormalizable, though renormalizability and other field theoretic constraints will not be explicitly considered in this work. (A review on field theory of hadrons can be found in Ref. 7 .)

A large number of theoretical attempts have been made to calculate the equation of state at low and high densities by using nonrelativistic two-body potentials adjusted to fit the experimental nucleon-nucleon scattering data. These calculations involve a large uncertainty as to the type of origin of interaction to be used. For example, the importance of the three-body interaction is receiving new attention. These calculations make it difficult to see possible simple interrelations between physically interesting quantities.

For example, in self-consistent relativistic mean-field models the influence of the compression constant $K\left(\rho_{0}\right)$ depends strongly on the effective nucleon mass in the ground state $m^{*}\left(\rho_{0}\right)$ as we will show below. For decreasing effective ground-state mass $m^{*}\left(\rho_{0}\right)$ the vector coupling constant $C_{v}$ is increasing and therefore the compression constant $K\left(\rho_{0}\right)$ becomes less and less important for the high-density behavior of the equation of state, while $m^{*}\left(\rho_{0}\right)$ becomes more influencial.

The aim of the present work is to explore explicitly these interconnections in a self-consistent relativistic field theory. ${ }^{8,9}$ This topic has been touched upon before. ${ }^{6}$ The theory allows for nuclear interactions that are not strictly of two-body Yukawa type but has therefore the disadvantage of a mostly unbounded scalar potential, ${ }^{6}$ as in particular if one fits the coupling constants of the mean-field theory to finite nuclei. ${ }^{10}$ This unbounded scalar potential leads to a very strong restriction for the possible sets of coupling constants.

\section{THE RELATIVSTIC MEAN-FIELD THEORY}

The nucleon field $\psi$ is assumed to interact with a scalar field $\varphi$ and a vector field $V_{\mu}$ through the following wellknown Lagrangian ${ }^{6-9}$

$$
\begin{aligned}
L= & \bar{\psi}\left(i \gamma_{\mu} \partial^{\mu}-m\right) \psi-U(\varphi) \\
& -\frac{1}{2} \partial_{\mu} \varphi \partial^{\mu} \varphi-\frac{1}{4} F_{\mu v} F^{\mu v}-\frac{1}{2} m_{v}^{2} V_{\mu} V^{\mu} \\
& -g_{s} \bar{\psi} \psi \varphi-g_{v} \bar{\psi} \gamma_{\mu} \psi V^{\mu} .
\end{aligned}
$$

The potential function $U(\varphi)$ is taken to be quartic polynomial in the field $\varphi .^{6,9}$ The theory is perturbatively renormalizable,

$$
U(\varphi)=\frac{1}{2} m_{s}^{2} \varphi^{2}+\frac{1}{3} b \varphi^{3}+\frac{1}{4} c \varphi^{4} .
$$

In the above expression the coefficient $c$, strictly speak- 
TABLE I. Twelve different sets of coupling constants are showed for fixed binding energy $E / A\left(\rho_{0}\right)=-16 \mathrm{MeV}$ at the ground-state density $\rho_{0}=0.15 / \mathrm{fm}^{3}$, while the ground-state compressibility $K\left(\rho_{0}\right)=210,300$, and $400 \mathrm{MeV}$ and the corresponding effective nucleon mass $m^{*}\left(\rho_{0}\right)=0.55,0.65,0.75$, and 0.85 are varied.

\begin{tabular}{lccccc}
\hline \hline & $C_{s}^{2}$ & $C_{v}^{2}$ & $B$ & $C$ & $m^{*}\left(\rho_{0}\right)$ \\
\hline$K\left(\rho_{0}\right)=400 \mathrm{MeV}$ & 367.152 & 264.687 & $-0.96713-3$ & $-0.13325-2$ & 0.55 \\
& 289.845 & 199.373 & $-0.54849-3$ & $+0.11485-2$ & 0.65 \\
& 209.830 & 132.497 & $+0.24501-2$ & $+0.19294-1$ & 0.75 \\
$K\left(\rho_{0}\right)=300 \mathrm{MeV}$ & 102.444 & 64.545 & $+0.68559-1$ & +0.42344 & 0.85 \\
& 380.792 & 264.687 & $-0.16175-2$ & $-0.22961-2$ & 0.55 \\
& 306.701 & 199.373 & $-0.21738-2$ & $-0.19474-2$ & 0.65 \\
$K\left(\rho_{0}\right)=210 \mathrm{MeV}$ & 233.239 & 132.497 & $-0.32898-2$ & $+0.39878-2$ & 0.75 \\
& 138.976 & 64.545 & $+0.17239-1$ & +0.19535 & 0.85 \\
& 393.169 & 264.687 & $-0.21687-2$ & $-0.31126-2$ & 0.55 \\
& 322.254 & 199.373 & $-0.35227-2$ & $-0.45166-2$ & 0.65 \\
& 255.909 & 132.497 & $-0.78475-2$ & $-0.81661-2$ & 0.75 \\
& 183.683 & 64.545 & $-0.17788-1$ & $+0.39674-1$ & 0.85 \\
\hline \hline
\end{tabular}

ing, should be positive, to assure the existence of a lower bound for the energy. We will allow $c$ to be a free parameter and determine its value from a phenomenological fit. As will be shown most of the fits are obtained for $c<0$. We will comment extensively on this point below.

For rotationally and translationally invariant symmetric nuclear matter, the field equations for the mesons in the mean-field approximation are

$$
\begin{aligned}
& m_{s}^{2} \varphi_{0}+b \varphi_{0}^{2}+c \varphi_{0}^{3}=-g_{s} \rho_{s}, \\
& m_{v}^{2} V_{0}=g_{v} \rho_{v}, \quad \mathbf{V}=0 .
\end{aligned}
$$

The energy density, the pressure, and the compressibility are given by

$$
\begin{aligned}
\epsilon= & \frac{1}{2} \frac{\boldsymbol{g}_{v}^{2}}{m_{v}^{2}} \rho_{B}^{2}+U\left(\varphi_{0}\right) \\
& +\frac{\gamma}{(2 \pi)^{3}} \int_{0}^{\infty} d^{3} k\left(k^{2}+m^{* 2}\right)^{1 / 2}[n(T)+\bar{n}(T)], \\
P= & \rho_{B}^{2} \frac{\partial}{\partial \rho_{B}} \frac{\epsilon}{\rho_{B}}, \quad K=9 \frac{\partial}{\partial \rho_{B}} P,
\end{aligned}
$$

while $m^{*}$ is defined as $m^{*}=m+g_{s} \varphi_{0}$. In the following $m^{*}$ will be meant always in units of the nucleon mass $m$ $(=939 \mathrm{MeV})$. The degeneracy factor $\gamma=4$ corresponds to spin-isospin- $\frac{1}{2}$ particles, while the effective chemical potential is defined as $v=\mu-g_{V} V_{0}$.

For cold nuclear matter the Fermi-Dirac distributions $n(T)$ and $\bar{n}(T)$ vanish and the integrals above can be solved analytically. The relevant parameters of the model are the dimensionless coupling constants

$$
\begin{aligned}
& C_{s}=\frac{g_{s} m}{m_{s}}, \quad C_{v}=\frac{g_{v} m}{m_{v}}, \\
& B=\frac{b}{g_{s}^{3} m}, \quad C=\frac{c}{g_{s}^{4}} .
\end{aligned}
$$

\section{DETERMINING THE COUPLING CONSTANTS}

These four coupling constants can be adjusted by fitting the four ground-state properties. The first step in doing this is to calculate the vector coupling constant $C_{v}$ for every value of $m^{*}\left(\rho_{0}\right)$. The point is mainly that due to the Hugenholtz-van Hove theorem ${ }^{11}$ the Fermi energy

TABLE II. Twelve different sets of coupling constants are showed for fixed binding energy $E / A\left(\rho_{0}\right)=-16 \mathrm{MeV}$ at the ground-state density $\rho_{0}=0.15 / \mathrm{fm}^{3}$, while the ground-state compressibility $K\left(\rho_{0}\right)=100,150$, and $180 \mathrm{MeV}$ and the corresponding effective nucleon mass $m^{*}\left(\rho_{0}\right)$ (cf. Table I) are varied.

\begin{tabular}{lccccc}
\hline \hline & $C_{s}^{2}$ & \multicolumn{1}{c}{$C_{v}^{2}$} & $B$ & $C$ & $m^{*}\left(\rho_{0}\right)$ \\
\hline$K\left(\rho_{0}\right)=180 \mathrm{MeV}$ & 397.316 & 264.687 & $-0.23457-2$ & $-0.33748-2$ & 0.55 \\
& 327.521 & 199.373 & $-0.39504-2$ & $-0.53314-2$ & 0.65 \\
& 263.833 & 132.497 & $-0.92559-2$ & $-0.11922-1$ & 0.75 \\
$K\left(\rho_{0}\right)=150 \mathrm{MeV}$ & 202.181 & 64.545 & $-0.27750-1$ & $-0.46026-2$ & 0.85 \\
& 401.474 & 264.687 & $-0.25194-2$ & $-0.36322-2$ & 0.55 \\
& 332.830 & 199.373 & $-0.43678-2$ & $-0.61265-2$ & 0.65 \\
$K\left(\rho_{0}\right)=100 \mathrm{MeV}$ & 271.952 & 132.497 & $-0.10614-1$ & $-0.15543-1$ & 0.75 \\
& 223.045 & 64.545 & $-0.37003-1$ & $-0.45728-1$ & 0.85 \\
& 408.427 & 264.687 & $-0.28021-2$ & $-0.40510-2$ & 0.55 \\
& 341.772 & 199.373 & $-0.50417-2$ & $-0.74099-2$ & 0.65 \\
& 285.934 & 132.497 & $-0.12772-1$ & $-0.21297-1$ & 0.75 \\
& 264.473 & 64.545 & $-0.51049-1$ & -0.10816 & 0.85 \\
\hline \hline
\end{tabular}


must be equal to the energy per baryon at saturation. That means

$$
E_{F}\left(\rho_{0}\right)=\frac{\epsilon}{\rho}\left(\rho_{0}\right)=\frac{E}{A}\left(\rho_{0}\right)+m
$$

with the Fermi energy $E_{F}=g_{v} V_{0}+\left(k_{F}^{2}+m^{* 2}\right)^{1 / 2}$.

Thus we see that $m^{*}\left(\rho_{0}\right)$ is a well-defined function of $C_{v}$ for fixed binding energy $E / A\left(\rho_{0}\right)$ at a given groundstate density $\rho_{0}$ and does not depend on the scalar coupling constants $C_{s}, b, c$ (Ref. 6). This means, in addition, that $C_{v}$ does not depend on the ground-state compressibility $K\left(\rho_{0}\right)$. This can be seen clearly in Tables I and II. From Eq. (6) it follows that for decreasing effective mass $m^{*}\left(\rho_{0}\right)$ the vector coupling constant $C_{v}$ increases (cf. Tables I and II). Therefore, the vector field becomes even more important at higher compression. Hence the largest influence of the vector field and therefore the stiffest equation of state is obtained for the lowest effective ground-state mass $m^{*}\left(\rho_{0}\right)$.

The three other constants are now adjusted to get saturation at ground state: $\rho_{0}=0.15 / \mathrm{fm}^{3}$ with a depth of $-16 \mathrm{MeV}$, so that compression constants between 100 and $400 \mathrm{MeV}$ are obtained. From $m^{*}\left(\rho_{0}\right) \leq 0.55$ or $K\left(\rho_{0}\right) \leq 180 \mathrm{MeV}$ the quartic scalar coupling constant $c$ is found to be negative.

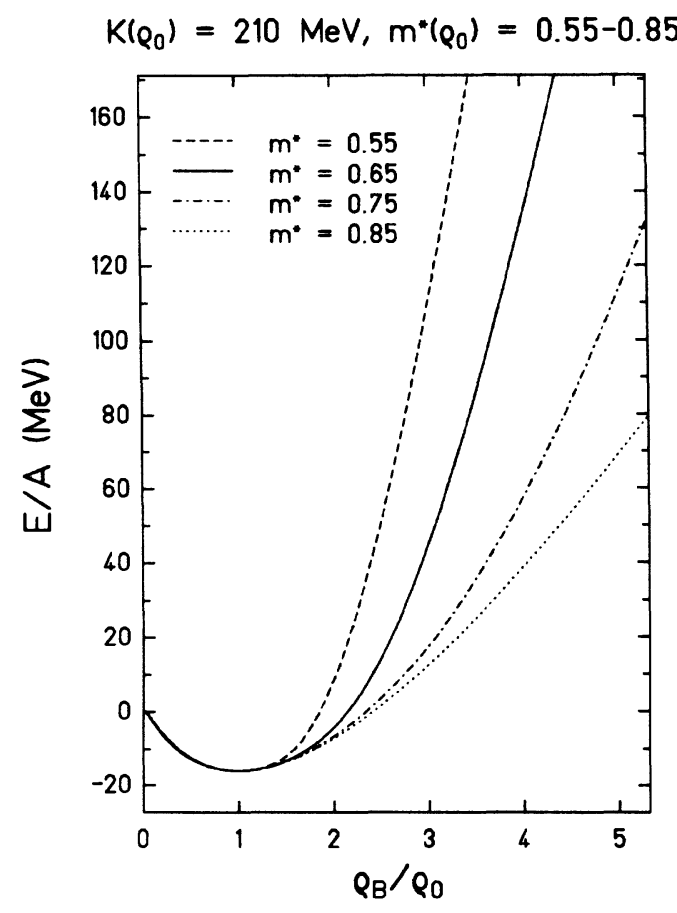

FIG. 1. The EOS for cold nuclear matter, that means binding energy per nucleon vs the baryon density, is shown for fixed ground-state binding energy $(E / A=-16 \mathrm{MeV})$, baryon density $\left(\rho_{0}=0.15 / \mathrm{fm}^{3}\right)$, and ground-state compressibility $K\left(\rho_{0}\right)=210$ $\mathrm{MeV}$, but for different effective masses $m^{*}\left(\rho_{0}\right)=0.55,0.65,0.75$, and 0.85 .

\section{HIGH-DENSITY BEHAVIOR OF THE EQUATION OF STATE}

At first we study again the dependence of the EOS, especially its high-density behavior, on the ground-state properties. $^{6}$ The strong dependence of the EOS on $m^{*}\left(\rho_{0}\right)$ is shown in Fig. 1. This effect is also observed for $K\left(\rho_{0}\right)=300$ and $400 \mathrm{MeV}$. This point is easily understood: For higher densities the vector field is the dominant contribution to the energy density [Eq. (4)]. But the vector field is proportional to the vector coupling constant $C_{v}^{2}$ which is determined by the ground-state effective nucleon mass [Eq. (6) and Table I]. ${ }^{6}$ To what extent does the equation of state then depend on the ground-state compression constant? The answer is shown in Fig. 2 for stiff equations of state, i.e., $m^{*}\left(\rho_{0}\right)=0.55$ at $T=100 \mathrm{MeV}$ (for $T=0 \mathrm{MeV}$ the equations of state have the same slope, i.e., the same negligible difference). A stiffer equation of state is favored from analysis of heavy-ion collision data. ${ }^{1,12-16}$ The nonlinear mean-field theory shows that there is negligible dependence of the equation of state on the compression constant. $^{6}$

These results show the difficulty if one were to extract the EOS and in particular the ground-state compression constant from measured pion yields. ${ }^{15}$ This conclusion is also reached by other work ${ }^{17}$ which emphasizes that the

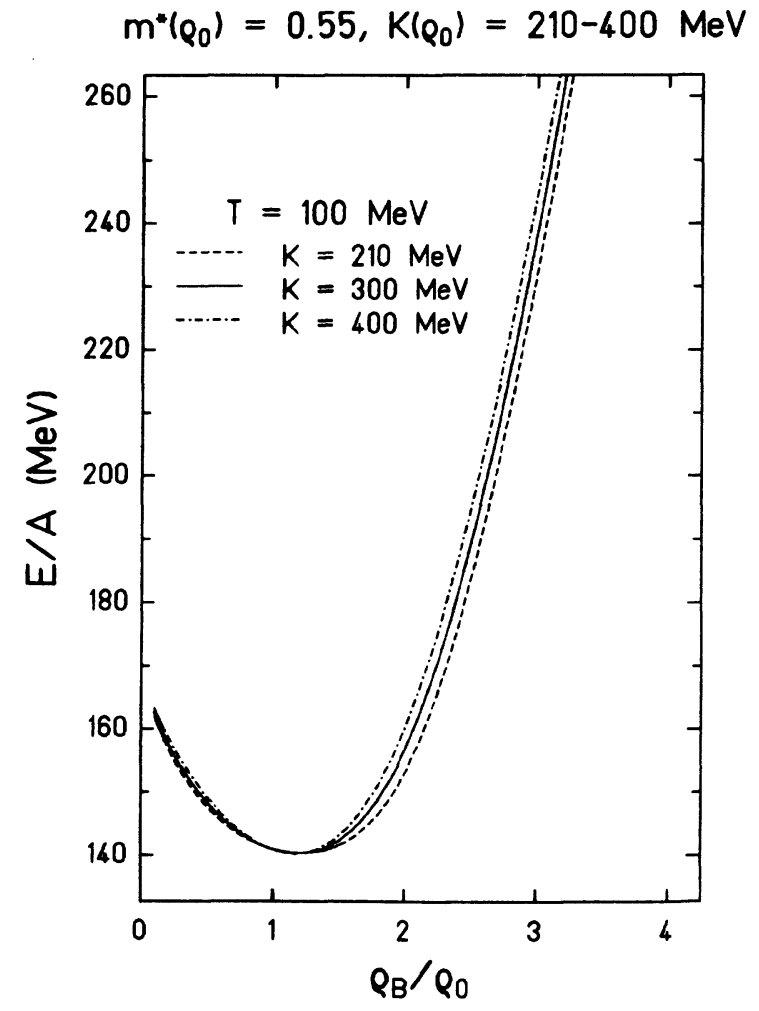

FIG. 2. The hot equation of state $(T=100 \mathrm{MeV})$ for a fixed value of the ground-state effective mass $m^{*}\left(\rho_{0}\right)=0.55$ and various values of the ground-state compressibility $K\left(\rho_{0}\right)=210,300$, and $400 \mathrm{MeV}$. 
ambiguities in the $\Delta$-coupling constants also yields large uncertainties in possible attempts to determine $E_{c}(\rho)$ from the pion data. ${ }^{15,16}$ The influence of the stiffness of the equation of state on the phase transition to quarkgluon plasma is studied elsewhere. ${ }^{18}$

\section{UNPHYSICAL BEHAVIOR OF THE MEAN-FIELD THEORY}

Let us consider now the equations of state with compression constants below $K\left(\rho_{0}\right)=200 \mathrm{MeV}$. The first remarkable point is that for all sets of coupling constants the quartic scalar coupling constant $c$ is always negative (see Table II and Fig. 3). The calculated equations of state for such compressibilities exhibit an unphysical behavior, cf. Figs. 4 and $5\left[K\left(\rho_{0}\right)=100\right.$ and $\left.150 \mathrm{MeV}\right]$. First of all observe the kinks in the EOS for $m^{*}\left(\rho_{0}\right)=0.75$ and 0.85 . There are two solutions (for $\left.0 \leq m^{*} \leq 1\right)$ up to a finite density. At higher density no solution of the self-consistent mean-field equation (3a) exists. The behavior results from the large, negative nonlinear terms. These bifurcations in the equation of state are obviously unphysical. This can also be observed by inspection of the velocity of first sound $c_{s}$ given by

$$
\left(c_{s} / c\right)^{2}=\frac{\partial P}{\partial \epsilon}=\frac{\partial P}{\partial \rho} \frac{\partial \rho}{\partial \epsilon},
$$

where $\partial \epsilon / \partial \rho$ is, for vanishing temperature, equal to the chemical potential $\mu$ and the Fermi energy $E_{F}$ [cf. Eq. (6)]. The result is shown in Fig. 6: The cusps in the equations of state correspond to poles in the sound velocity. This acausal behavior of the relativistic mean-field theory is also reflected in the effective mass (Fig. 7). For these sets of coupling constants the effective mass versus the baryon density turns backwards at $\rho_{B} \simeq 2 \rho_{0}$. Hence two

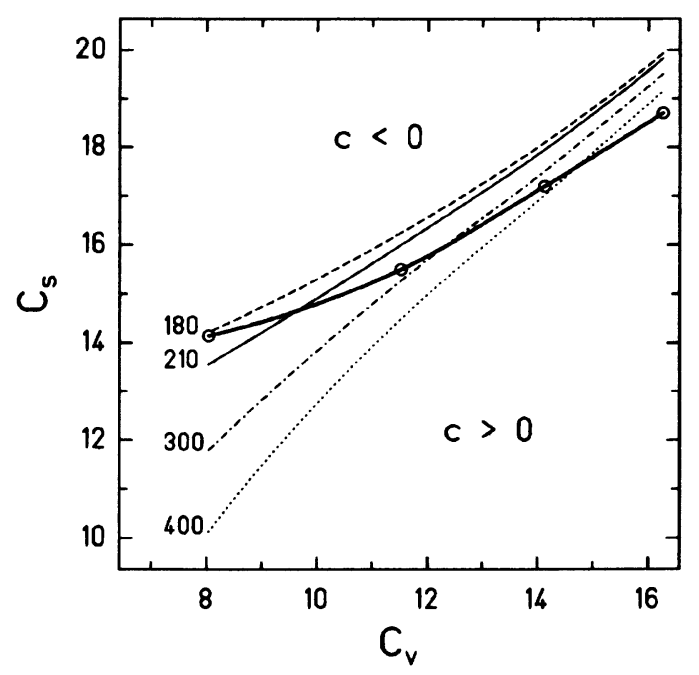

FIG. 3. The dependence of the scalar coupling constant $C_{s}$ on the vector coupling constant $C_{v}$ is plotted for various compression constants $\left[K\left(\rho_{0}\right)=180,210,300\right.$, and $\left.400 \mathrm{MeV}\right]$. Also the border line between positive and negative value for the quartic scalar coupling constant $c$ is presented: $c=0$. The four open circles indicating the different effective ground-state masses $m^{*}\left(\rho_{0}\right)=0.85,0.75,0.65$, and 0.55 (from left to right).

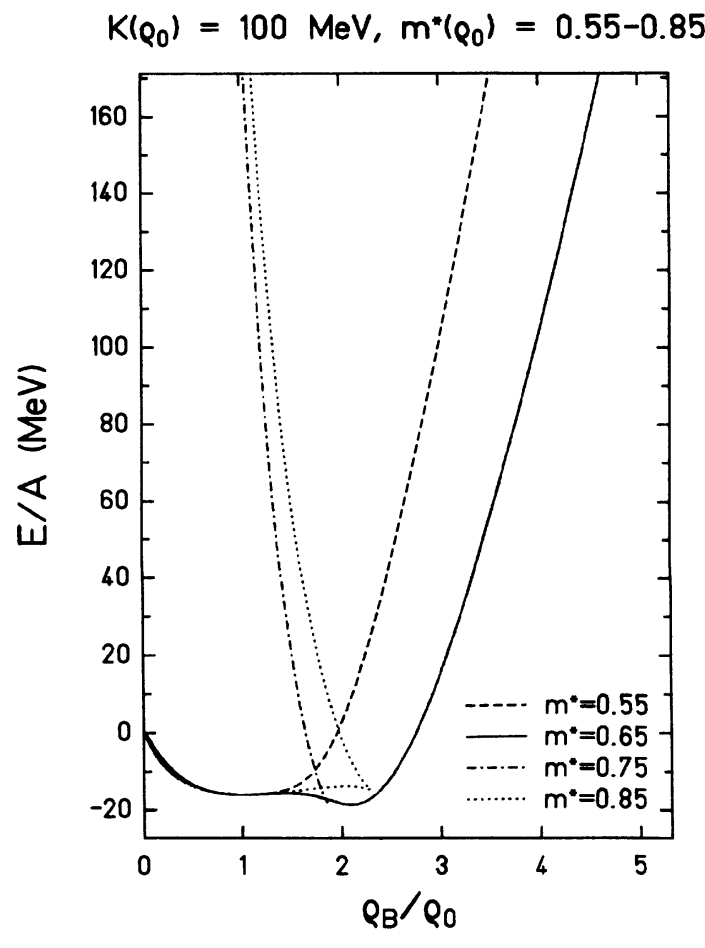

FIG. 4. The cold equation of state for a fixed value of the ground-state compressibility $K\left(\rho_{0}\right)=100 \mathrm{MeV}$ and various values for the effective mass: $m^{*}\left(\rho_{0}\right)=0.55,0.65,0.75$, and 0.85 .

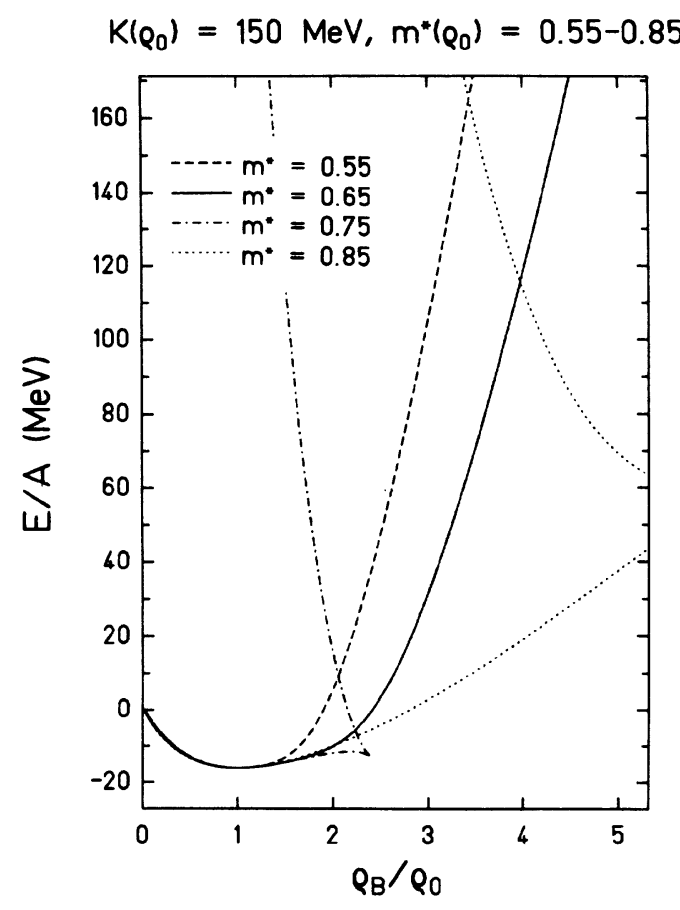

FIG. 5. The same equation of state as Fig. 5, but for $K\left(\rho_{0}\right)=150 \mathrm{MeV}$. 
solutions are found in a small density region and none for higher densities. One should mention that a third unstable solution can be found for effective masses higher than the normal nucleon mass.

For $K\left(\rho_{0}\right)=180 \mathrm{MeV}$ the two equations of state for the higher ground-state masses $\left[m^{*}\left(\rho_{0}\right)=0.75\right.$ and 0.85$]$ cross each other at $\rho_{B} \cong 1.75 \rho_{0}$ and $3.75 \rho_{0}$.

This acausal behavior is due to the problems with the scalar potential mentioned above. In Fig. 8 the negative of the scalar density $\left(-\rho_{s}\right)$, the scalar potential $U\left(\varphi_{0}\right)$, and the derivative $U^{\prime}\left(\varphi_{0}\right)$ are plotted for $K\left(\rho_{0}\right)=100$ $\mathrm{MeV}$ and $m^{*}\left(\rho_{0}\right)=0.75$. The solid line and the dashed line show the negative of the scalar density for $\rho_{B}=\rho_{0}$ and $\rho_{B}=4 \rho_{0}$, respectively, while the dashed-dotted line shows the first derivative of the scalar potential $U\left(\varphi_{0}\right)$, namely, $U^{\prime}\left(\varphi_{0}\right)$ plotted versus the effective nucleon mass $m^{*}$. For a fixed baryon density $\rho_{B}$ each crossing point of these two lines corresponds to a solution of the selfconsistency relation ( $3 a$ ) of the scalar field (or equivalently the effective nucleon mass):

$$
U^{\prime}\left(\varphi_{0}\right)=-g_{s} \rho_{s} \text {. }
$$

Figure 8 shows for $\rho_{B}=\rho_{0}=0.15 / \mathrm{fm}^{3}$ three crossing points, i.e., three solutions (one for $m^{*}>1$ ). The same scenario is plotted for a higher density: $\rho_{B}=4 \rho_{0}$. It shows that the scalar density $\rho_{s}$ increases, while the

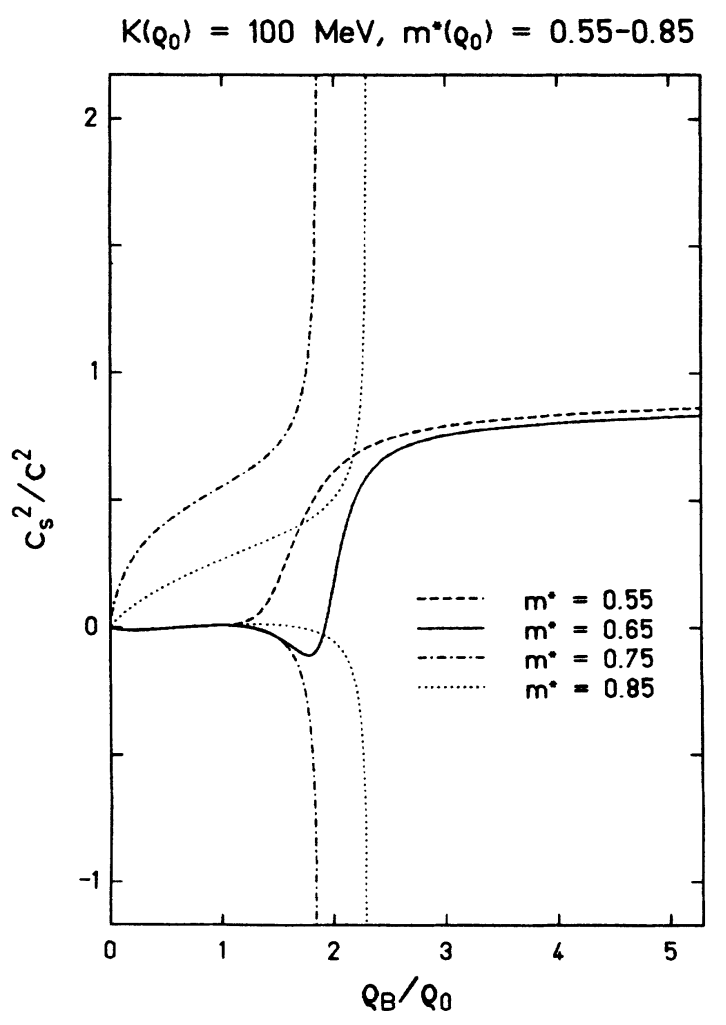

FIG. 6. For the equation of state of Fig. 4 with $m^{*}\left(\rho_{0}\right)=0.75$ the square of the first sound velocity is plotted vs the compres$\operatorname{sion} \rho_{B} / \rho_{0}$.

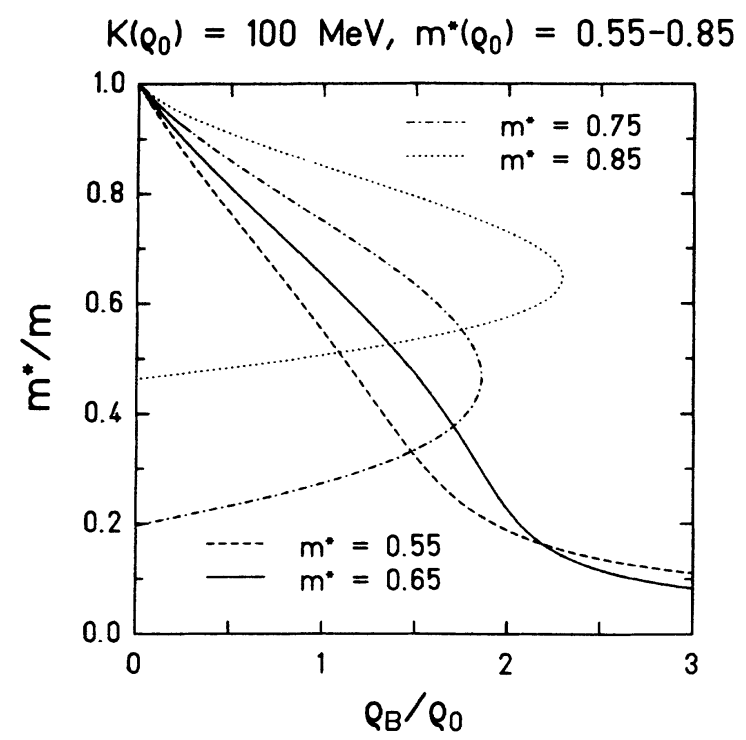

FIG. 7. For the equation of state of Fig. 4 with $m^{*}\left(\rho_{0}\right)=0.75$ the effective nucleon mass is plotted vs the compression $\rho_{B} / \rho_{0}$.

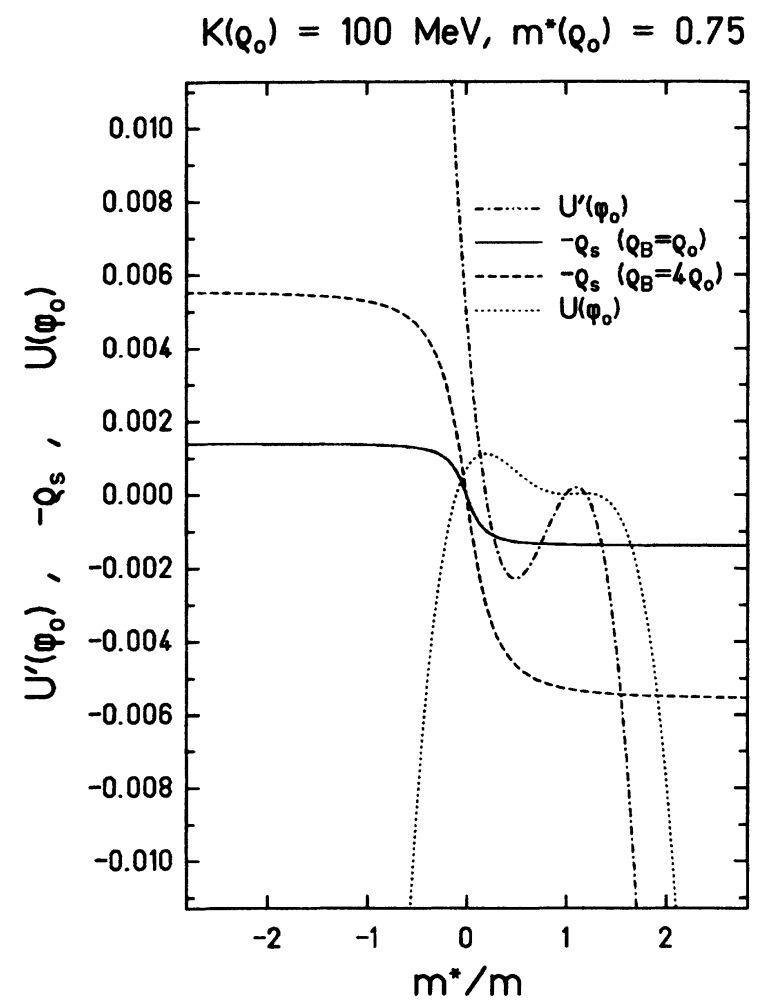

FIG. 8. For the equation of state of Fig. 4 with $m *\left(\rho_{0}\right)=0.75$ the negative of the scalar density $-\rho_{s}$, the first derivative of the scalar potential $U\left(\varphi_{0}\right)$, namely, $U^{\prime}\left(\varphi_{0}\right)$ (dashed-dotted line) and the scalar potential itself (dotted line) is plotted vs the effective nucleon mass $m^{*}$ for the ground-state density $\rho_{0}=0.15 / \mathrm{fm}^{3}$ (full line) and for $\rho_{B}=4 \rho_{0}$ (dashed line). 
derivative of the scalar potential does not change because it is independent of $\rho_{B}$. Therefore, only the unstable solution with $m^{*}>1$ is found. The strongly negative quartic coupling constant $c$ causes the unbounded scalar potential $U\left(\varphi_{0}\right)$ (dotted line) with a shallow dip in the derivative $U^{\prime}\left(\varphi_{0}\right)$. A stable solution of the selfconsistency relation (8) requires a minimum in $U\left(\varphi_{0}\right)$. Otherwise this state is unstable (quantum fluctuations are neglected in the mean field approximation). In our case $\left[K\left(\rho_{0}\right)=100 \mathrm{MeV}\right.$ and $\left.m^{*}\left(\rho_{0}\right)=0.75\right]$ the minimum is very shallow (the depth is below $1 \mathrm{MeV}$ ). But also for higher ground-state compression constants $K\left(\rho_{0}\right) \leq 270$ $\mathrm{MeV}$ [e.g., $K\left(\rho_{0}\right)=210 \mathrm{MeV}$, Fig. 9] a shallow minimum is obtained, although it is causal and does not show any irregularities (compare Fig. 1). This behavior does not depend on the ground-state density. We checked it for $\rho_{0}=0.16$ and $0.17 \mathrm{fm}^{-3}$. Therefore, the best fit set of coupling constants from Reinhard et al. (Ref. 10) is not stable, as well as all other sets of coupling constants with a negative $c$, such as that predicted by the first of Refs. 6 . We also found that for compression constants below $K\left(\rho_{0}\right)=150 \mathrm{MeV}$ abnormal, density isomeric states [e.g., in Fig. 4 for $K\left(\rho_{0}\right)=100 \mathrm{MeV}$ and $\left.m^{*}\left(\rho_{0}\right)=0.65\right]$ can occur at $\rho=2 \rho_{0}$ and $E / A \cong-20 \mathrm{MeV}$, i.e., below the normal ground state of nuclear matter. This second minimum is observed while the values of the binding energy per nucleon, the ground-state density, compressibility, and the effective mass of the nucleon in the normal state are correctly reproduced. Boguta and Bodmer ${ }^{9}$ did not obtain any density isomer in spite of explicitly searching for it. The too high ground-state density $\left(\rho_{0}=0.194\right)$ they use could be a reason for it.

At this point we want to emphasize that the abnormalities discussed above have no connection to the phase transitions that have been discussed in the literature, e.g., the liquid vapor phase transition, ${ }^{19,20}$ the possible phase transition to delta matter ${ }^{20,21}$ and the transition to a baryonic plasma. ${ }^{20,22,23}$

\section{SUMMARY}

We have observed that the relativistic nonlinear meanfield theory for symmetric nuclear matter is rather restrictive when applied to ground-state compressibilities below $K\left(\rho_{0}\right)=200 \mathrm{MeV}$. Cusps, acausal behavior $\left(c_{s}>1\right)$, and secondary minima appear for $m^{*}\left(\rho_{0}\right) \simeq 0.8$ and $m^{*}\left(\rho_{0}\right)=0.65$, respectively. The problematic compression constants are dangerously close to the sometimes used values: $K\left(\rho_{0}\right)=210 \pm 30 \mathrm{MeV} .^{3}$

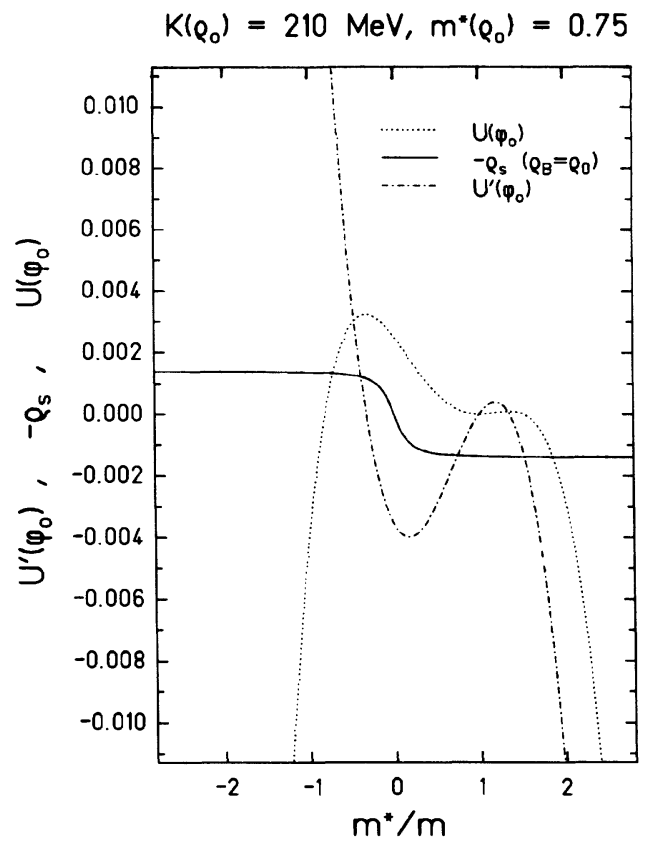

FIG. 9. For the equation of state of Fig. 1 with $m^{*}\left(\rho_{0}\right)=0.75$ the negative of the scalar density $-\rho_{s}$, the scalar potential $U\left(\varphi_{0}\right)$ (dotted line) and the first derivative (dashed-dotted line) are plotted vs the effective nucleon mass $\mathrm{m}^{*} / \mathrm{m}$ for the groundstate density $\rho_{0}=0.15 / \mathrm{fm}^{3}$ (full line).

For higher compressibilities, $K\left(\rho_{0}\right)>200 \mathrm{MeV}$, the relativistic mean-field theory seems to provide a convenient framework for discussing the equation of state. In particular, we have seen that $m^{*}\left(\rho_{0}\right)$ and not $K\left(\rho_{0}\right)$ is the decisive quantity which yields the high-density behavior of the nuclear equation of state. However, also here several of the selected sets of coupling constants possess an unbound scalar potential $U\left(\varphi_{0}\right)$, i.e., $c<0$ (Table I). So it is not possible to fit finite nuclei as best as you can ${ }^{10}$ and simultaneously get a stable EOS with a bounded scalar potential. All parameter sets with $c<0$ must be rejected.

\section{ACKNOWLEDGMENTS}

We would like to thank John Boguta and Norman K. Glendenning for valuable discussions. This work was supported by Bundesministerium für Forschung und Technologie and Gesellschaft für Schwerionenforschung.
${ }^{1}$ H. Stöcker and W. Greiner, Phys. Rep. 137, 277 (1986).

${ }^{2}$ W. D. Myers and W. J. Swiatecki, Ann. Phys. 55, 395 (1967).

3J.-P. Blaizot, D. Gogny, and B. Grammaticos, Nucl. Phys. A265, 315 (1976).

${ }^{4}$ G. E. Brown and E. Osnes, Phys. Lett. 159B, 223 (1985).

${ }^{5}$ G. Co' and J. Speth, Phys. Rev. Lett. 57, 547 (1986). Comment on preceding paper: A. van der Woude, W. T. A. Borghols, S. Brandenburg, M. M. Sharma, and M. N. Harakeh, Phys. Rev. Lett. 58, 2383 (1987). Response to the comment: G. Co' and J. Speth, ibid. 58, 2384 (1987).

6J. Boguta and H. Stöcker, Phys. Lett. 120B, 289 (1983); J. Boguta, ibid. 106B, 250 (1981). 
${ }^{7}$ B. Serot and J. D. Walecka, Adv. Nucl. Phys. 16 (1986).

${ }^{8}$ J. D. Walecka, Ann. Phys. 83, 491 (1974).

${ }^{9}$ J. Boguta and A. R. Bodmer, Nucl. Phys. 292A, 413 (1977).

${ }^{10}$ P.-G. Reinhard, M. Rufa, J. Maruhn, W. Greiner, and J. Friedrich, Z. Phys. A 323, 13 (1986).

${ }^{11}$ N. M. Hugenholtz and L. van Hove, Physica 24, 363 (1958). V. F. Weisskopf, Nucl. Phys. 3, 423 (1957).

12J. Aichelin, G. Peilert, A. Rosenhauer, H. Stöcker, and W. Greiner, Phys. Rev. Lett. 58, 1926 (1987).

${ }^{13}$ J. J. Molitoris, D. Hahn, and H. Stöcker, Nucl. Phys. A447, 13 (1985). J. J. Molitoris and H. Stöcker, Phys. Rev. C 32, 346 (1985).

${ }^{14} \mathrm{~J}$. Boguta and H. J. Crawford (to be published); J. Boguta, Report (unpublished).

${ }^{15}$ J. W. Harris, R. Stock, R. Bock, R. Brockmann, A. Sandoval, H. Ströbele, G. Odyniec, H. G. Pugh, L. S. Schröder, R. E. Renfordt, D. Schall, D. Bangert, W. Rauch, and K. L. Wolf, Phys. Lett. 153B, 377 (1985); R. Stock, Phys. Rep. 135, 259 (1986); J. W. Harris, G. Odyniec, H. G. Pugh, L. S. Schröder, M. L. Tincknell, W. Rauch, R. Stock, R. Bock, R. Brock- mann, A. Sandoval, H. Ströbele, R. E. Renfordt, D. Schall, D. Bangert, J. P. Sullivan, K. L. Wolf, A. Dacal, C. Guerra, and M. E. Ortiz, Phys. Rev. Lett. 58, 463 (1987).

${ }^{16}$ H. Stöcker, W. Greiner, and W. Scheid, Z. Phys. A 286, 121 (1978); D. Hahn and H. Stöcker, Nucl. Phys. A452, 723 (1986).

${ }^{17}$ B. M. Waldhauser, J. A. Maruhn, H. Stöcker, and W. Greiner, Z. Phys. A 328, 19 (1987).

${ }^{18}$ B. M. Waldhauser, D. H. Rischke, B. L. Friman, and W. Greiner, Report (unpublished).

${ }^{19}$ I. Lovas and Gy. Wolf, Acta Phys. Hung. 58, 23 (1985).

${ }^{20}$ B. M. Waldhauser, J. Theis, J. A. Maruhn, H. Stöcker, and W. Greiner, Phys. Rev. C 36, 1019 (1987).

${ }^{21}$ J. Boguta, Phys. Lett. 109B, 251 (1982).

${ }^{22}$ J. Theis, G. Graebner, G. Buchwald, J. A. Maruhn, W. Greiner, H. Stöcker, and J. Polonyi, Phys. Rev. D 28, 2286 (1983).

${ }^{23}$ N. K. Glendenning, Phys. Lett. 144B, 158 (1984); 185B, 275 (1987); Nucl. Phys. A469, 600 (1987). 